\title{
The background scale Ward identity in quantum gravity
}

\author{
Roberto Percacci $^{1,2, a}$, Gian Paolo Vacca ${ }^{3, b}$ \\ ${ }^{1}$ International School for Advanced Studies, via Bonomea 265, 34136 Trieste, Italy \\ 2 INFN, Sezione di Trieste, Trieste, Italy \\ ${ }^{3}$ INFN, Sezione di Bologna, via Irnerio 46, 40126 Bologna, Italy
}

Received: 28 November 2016 / Accepted: 11 January 2017 / Published online: 27 January 2017

(C) The Author(s) 2017. This article is published with open access at Springerlink.com

\begin{abstract}
We show that with suitable choices of parametrization, gauge fixing and cutoff, the anomalous variation of the effective action under global rescalings of the background metric is identical to the derivative with respect to the cutoff, i.e. to the beta functional, as defined by the exact RG equation. The Ward identity and the RG equation can be combined, resulting in a modified flow equation that is manifestly invariant under global background rescalings.
\end{abstract}

\section{Introduction}

One of the most vexing challenges facing the asymptotic safety approach to quantum gravity has been the double dependence of the effective action on two fields, the background metric and the fluctuation field. It is only when both dependences are taken into account that one can write an exact flow equation [1]. On the other hand, physical results should be largely independent of the choice of background. In fact, at the classical level, the action is invariant under simultaneous transformations of the background and fluctuation. At the simplest level, when one uses a linear parametrization,

$g_{\mu \nu}=\bar{g}_{\mu \nu}+h_{\mu \nu}$,

these are just the shift transformations

$\delta \bar{g}_{\mu \nu}=\epsilon_{\mu \nu}, \quad \delta h_{\mu \nu}=-\epsilon_{\mu \nu}$.

Ideally the effective action should also be invariant under the same transformations. However, the background gaugefixing procedure and the addition of a cutoff term in the action spoil this invariance. In much (in practice, up to 2008, all) work on asymptotic safety this issue has been avoided by

\footnotetext{
a e-mail: percacci@sissa.it

be-mail: vacca@bo.infn.it
}

restricting oneself to the so-called "single-field approximation" where one sets the fluctuation field to zero. The dangers of considering only the background dependence had been pointed out in $[2,3]$. In the last few years there have been made several efforts to address this issue.

One is to study bi-metric truncations [4-6] and impose shift-invariance only in the IR limit [7]. If one were able to calculate the whole bi-metric flow, then the background flow could be obtained by setting the classical fields to zero. Thus in practice one method to improve on single-field truncations is to keep as much as possible of the fluctuation dependence, by calculating the flow of the two-, three- and possibly fourpoint functions of the fluctuation [8-12]. Alternatively one can try to solve simultaneously the Ward identity and the flow equation. This could be achieved in the conformally reduced case [13-15]. Other related ideas have been discussed in [1618].

A step forward has recently been made by Morris for the special case when $\epsilon_{\mu \nu}=2 \epsilon \bar{g}_{\mu \nu}$, i.e. when the background is simply rescaled by a constant factor [19]. He derived the modified Ward identity for this transformation and showed that in six dimensions the anomalous terms coming from the cutoff have the same form as the RG equation. In this way the Ward identity and the RG equation can be combined in a single equation that is amenable to explicit treatment by the methods that are in current use. The drawback of the proposed procedure is that it only seems to work in six dimensions.

We show in this paper that modifying some steps of the procedure is sufficient to obtain the same result in any dimension. The first and most crucial step is the replacement of the linear split (1.1) by the exponential parametrization

$g_{\mu \nu}=\bar{g}_{\mu \rho}\left(e^{\mathbf{X}}\right)_{\nu}^{\rho}$,

where

$X^{\rho}{ }_{\nu}=\bar{g}^{\rho \sigma} h_{\sigma \nu}$. 
This parametrization is widely used in two-dimensional quantum gravity [20-22]. It has been introduced in the functional RG setting in $[23,24]$. Its general virtues have been further discussed in [25-31], and it has been employed in several other explicit calculations [32-36].

The second step is to make sure that no dimensionful parameter enters the gauge-fixing term. In the EinsteinHilbert truncation it is convenient and customary to have a prefactor $Z_{N}=1 /(16 \pi G)$, so that the gauge-fixing terms combine smoothly with the Hessian, but this introduces and unnecessary and, as we shall see, unwanted breaking of background scale invariance. We will use a higher-derivative gauge fixing, which amounts to introducing some power of the Laplacian in the gauge-fixing term. This type of gauge fixing is often used with four-derivative gravitational actions [37-40] but normally not in the Einstein-Hilbert truncation. There is, however, no fundamental reason for this, other than simplicity [31].

The third step is to similarly avoid dimensionful parameters in the cutoff term, except for the cutoff scale itself. We will use a "pure" cutoff, namely one that does not contain any running parameter $[33,41]$. As with the gauge-fixing term, in the Einstein-Hilbert truncation it is convenient to have a prefactor $1 /(16 \pi G)$. In the $f(R)$ truncation the corresponding prefactor is $-f^{\prime}(R)$. This dependence of the cutoff on running couplings is, however, the source of unnecessary anomalies.

We will see that, with these choices, the gauge fixing becomes invariant and the anomalous terms in the Ward identity coming from the cutoff have the same form as the RG equation. Then the Ward identity expresses the invariance of the effective action under the transformation of the background, fluctuation and a simultaneous rescaling of the cutoff scale. This identity can be solved and results simply in the definition of new variables that are invariant under background scale transformations. The RG equation, written in these variables, no longer depends on the scale of the background metric and has the same form as the flow equation that is commonly used. Although for the time being limited to simple scalings, this points toward a practical solution of the background-field dependence.

In Sect. 2 we discuss the transformation of the fields and of the gauge-fixing and cutoff actions. In Sect. 3 we derive the Ward identity and combine it with the RG equation. Section 4 contains a short discussion.

\section{Variations}

\subsection{Fields}

In this section we will often suppress indices and treat twoindex tensors as matrices. Thus (1.3) and (1.4) will be written $\mathbf{g}=\overline{\mathbf{g}} e^{\mathbf{X}}, \quad \mathbf{X}=\overline{\mathbf{g}}^{-1} \mathbf{h}$

(Normally one would denote also $X$ by the symbol $h$, but this would give rise to ambiguities when indices are suppressed.) Note that $X$ is a linear map of the tangent space to itself, so powers of $X$ and the trace of $X$ do not require use the metric and are basis-independent.

Our first task is the following: given an infinitesimal transformation $\delta \overline{\mathbf{g}}$ of the background metric, find a transformation $\delta \mathbf{h}$ of the fluctuation field such that the full metric $\mathbf{g}$ is invariant. We must have

$0=\delta \mathbf{g}=\delta \overline{\mathbf{g}} e^{\mathbf{X}}+\overline{\mathbf{g}} \delta e^{\mathbf{X}}$.

We use in (2.1) the relation

$\delta e^{\mathbf{X}} e^{-\mathbf{X}}=\frac{e^{a d \mathbf{x}}-1}{a d_{\mathbf{X}}} \delta \mathbf{X}$

where $a d_{\mathbf{X}} \mathbf{Y}=[\mathbf{X}, \mathbf{Y}]$, and the relation $e^{\mathbf{X}} \mathbf{Y} e^{-\mathbf{X}}=e^{a d_{\mathbf{X}}} \mathbf{Y}$, to obtain

$\delta \mathbf{X}=-\frac{a d \mathbf{X}}{e^{a d \mathbf{X}}-1} \overline{\mathbf{g}}^{-1} \delta \overline{\mathbf{g}}$.

Then, using Eq. (1.4), we derive the variation of $\mathbf{h}$ :

$\delta \mathbf{h}=\delta \overline{\mathbf{g}} \mathbf{X}+\overline{\mathbf{g}} \delta \mathbf{X}$.

Expanding

$\frac{a d_{\mathbf{X}}}{e^{a d_{\mathbf{X}}-1}}=1-\frac{a d_{\mathbf{X}}}{2}+\frac{a d_{\mathbf{X}}^{2}}{12}-\frac{a d_{\mathbf{X}}^{4}}{720}+\frac{a d_{\mathbf{X}}^{6}}{30240}-\cdots$

one could treat in this way general variations. Things, however, simplify drastically when we consider Weyl transformations

$\delta \bar{g}_{\mu \nu}=2 \epsilon \bar{g}_{\mu \nu}$,

where $\epsilon$ is an infinitesimal transformation parameter (a scalar function). In this case $\overline{\mathbf{g}}^{-1} \delta \overline{\mathbf{g}}=2 \epsilon \mathbf{1}$ is a multiple of the unit matrix, so $\delta \mathbf{X}=-2 \epsilon \mathbf{1}$, and thus using (2.4)

$\delta h_{\mu \nu}=2 \epsilon\left(h_{\mu \nu}-\bar{g}_{\mu \nu}\right)$.

It is convenient to decompose the fluctuation field into its tracefree and trace parts:

$h_{\mu \nu}=h_{\mu \nu}^{T}+\frac{1}{d} \bar{g}_{\mu \nu} h$

where $\bar{g}^{\mu v} h_{\mu \nu}^{T}=0$. We could further decompose the tracefree part into spin-two, spin-one and spin-zero parts, as in the York decomposition, but this is not necessary. The following 
considerations hold whether one uses the York decomposition or not.

We have

$\delta h_{\mu \nu}=\delta h_{\mu \nu}^{T}+\frac{1}{d} 2 \epsilon \bar{g}_{\mu \nu} h+\frac{1}{d} \bar{g}_{\mu \nu} \delta h$.

On the other hand inserting (2.8) in the r.h.s. of (2.7) and comparing the trace and tracefree parts we find

$$
\begin{aligned}
\delta h_{\mu \nu}^{T} & =2 \epsilon h_{\mu \nu}^{T}, \\
\delta h & =-2 d \epsilon .
\end{aligned}
$$

Note that the tracefree fluctuation transforms in the same way as the metric whereas the trace transforms purely by a shift. This is distinctly different from the behavior in the linear decomposition (1.1) and lies at the root of the subsequent simplifications. In the special case when the manifold is compact and $\epsilon$ is constant, we can be even more specific. If we decompose the trace into the constant part and its orthogonal complement

$h=\underline{h}+h^{\perp}$,

which is defined by the condition that its integral over the whole manifold is zero, the whole variation of $h$ is due to the constant component, while $h^{\perp}$ is invariant:

$\delta \underline{h}=-2 d \epsilon ; \quad \delta h^{\perp}=0$.

We observe that if we restrict ourselves from the beginning to Weyl transformations of the background metric, there is a more direct derivation of (2.10). Raising one index in (2.8) one can write $\mathbf{X}=\mathbf{X}^{T}+\mathbf{1} h / d$, where $\mathbf{X}^{T}$ is traceless. Therefore

$\mathbf{g}=\overline{\mathbf{g}} e^{h / d} e^{\mathbf{X}^{T}}$.

If the background metric undergoes the finite transformation $\overline{\mathbf{g}} \rightarrow \overline{\mathbf{g}} e^{2 \epsilon}$, invariance of the full metric can be maintained by the compensating transformation $h \rightarrow h-2 d \epsilon$, while $\mathbf{X}^{T}$ is left invariant. Then $\delta \mathbf{h}^{T}=\delta\left(\overline{\mathbf{g}} \mathbf{X}^{T}\right)=2 \epsilon \overline{\mathbf{g}} X^{T}=2 \epsilon \mathbf{h}^{T}$, which is just (2.10). In the following we restrict ourselves to constant Weyl transformations of the background metric.

\subsection{Gauge fixing}

Let us consider a gauge-fixing term

$S_{\mathrm{GF}}=\frac{1}{2 \alpha} \int d^{d} x \sqrt{\bar{g}} F_{\mu} Y^{\mu \nu} F_{\nu}$,

where $Y^{\mu v}$ is in general a differential operator,

$$
F_{\mu}=\bar{\nabla}_{\rho} h_{\mu}^{\rho}-\frac{\beta+1}{d} \bar{\nabla}_{\mu} h=\bar{\nabla}_{\rho} h_{\mu}^{T \rho}-\frac{\beta}{d} \bar{\nabla}_{\mu} h,
$$

and $\bar{\nabla}$ is the covariant derivative of $\bar{g}_{\mu \nu}$. Since the background Christoffel symbols are invariant under background rescalings, taking into account also the variation of the inverse metric that is hidden in $F_{\mu}$, one finds

$\delta F_{\mu}=0$.

Let $\bar{\Delta}$ be a second-order Laplace-type operator constructed with the background metric. It transforms under background rescalings as

$\delta \bar{\Delta}=-2 \epsilon \bar{\Delta}$.

The gauge-fixing term will be invariant under background rescalings if we choose

$Y^{\mu \nu}=\bar{\Delta}^{\frac{d-2}{2}} \bar{g}^{\mu \nu}$.

In order to derive the Faddeev-Popov operator, we start from the transformation of the full metric under an infinitesimal diffeomorphism $\eta$, which is given by the Lie derivative

$\delta_{\eta} g_{\mu \nu}=\mathcal{L}_{\eta} g_{\mu \nu} \equiv \nabla_{\mu} \eta_{\nu}+\nabla_{\nu} \eta_{\mu}$.

(Note that there are no bars on the $\nabla \mathrm{s}$ here.) As usual, we have to define transformations of $\bar{g}$ and $h$ that, used in (1.3), yield (2.19). The simplest one is the background transformation. We use again matrix notation as in the preceding section. If we treat $\overline{\mathbf{g}}$ and $\mathbf{X}$ as tensors under $\delta_{\eta}$, i.e.

$\delta_{\eta}^{(B)} \overline{\mathbf{g}}=\mathcal{L}_{\eta} \overline{\mathbf{g}} ; \quad \delta_{\eta}^{(B)} \mathbf{X}=\mathcal{L}_{\eta} \mathbf{X}$

then also $\delta_{\eta}^{(B)} e^{\mathbf{X}}=\mathcal{L}_{\eta} e^{\mathbf{X}}$ and (2.19) follows. By definition, the "quantum" gauge transformation of $\mathbf{X}$ is such as to reproduce (2.19) when $\overline{\mathbf{g}}$ is held fixed:

$\delta_{\eta}^{(Q)} \overline{\mathbf{g}}=0 ; \quad \overline{\mathbf{g}} \delta_{\eta}^{(Q)} e^{\mathbf{X}}=\mathcal{L}_{\eta} \mathbf{g}=\mathcal{L}_{\eta} \overline{\mathbf{g}} e^{\mathbf{X}}+\overline{\mathbf{g}} \mathcal{L}_{\eta} e^{\mathbf{X}}$.

From the latter relation we find

$e^{-\mathbf{X}_{\delta_{\eta}}(Q)} e^{\mathbf{X}}=e^{-\mathbf{X}}\left(\overline{\mathbf{g}}^{-1} \mathcal{L}_{\eta} \overline{\mathbf{g}}\right) e^{\mathbf{X}}+e^{-\mathbf{X}} \mathcal{L}_{\eta} e^{\mathbf{X}}$.

Then using (2.2) one finds

$\delta_{\eta}^{(Q)} \mathbf{X}=\frac{a d \mathbf{X}}{e^{a d_{\mathbf{X}}-\mathbf{1}}}\left(\overline{\mathbf{g}}^{-1} \mathcal{L}_{\eta} \overline{\mathbf{g}}+\mathcal{L}_{\eta} e^{\mathbf{X}} e^{-\mathbf{X}}\right)$.

The Fadeev-Popov operator, acting on a ghost field $C_{\mu}$, is defined by

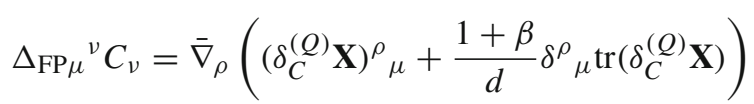


where the infinitesimal transformation parameter $\eta$ has been replaced by the ghost. The full ghost action then has the form [31]

$$
S_{\mathrm{gh}}\left(C_{\mu}^{*}, C_{\mu} ; \bar{g}_{\mu \nu}\right)=\int d^{d} x \sqrt{\bar{g}} C_{\mu}^{*} Y^{\mu \nu} \Delta_{\mathrm{FP} \nu}{ }^{\rho} C_{\rho} .
$$

Note that this action contains infinitely many interaction terms. Expanding (2.24) to first order in $\mathbf{X}$ we find ${ }^{1}$ :

$\delta_{C}^{(Q)} \mathbf{X}=\overline{\mathbf{g}}^{-1} \mathcal{L}_{C} \overline{\mathbf{g}}+\mathcal{L}_{C} \mathbf{X}+\frac{1}{2}\left[\overline{\mathbf{g}}^{-1} \mathcal{L}_{C} \overline{\mathbf{g}}, \mathbf{X}\right]+O\left(C \mathbf{X}^{2}\right)$.

In the single-metric truncation, where one puts $\mathbf{X}=0$ from the start, the Faddeev-Popov operator is determined by the first term in this expansion. It is a (generally non-minimal) Laplace-type operator constructed with the background metric, and therefore transforms as in (2.17). Invariance under global Weyl rescalings can be achieved simply demanding

$\delta C_{\mu}^{*}=0, \quad \delta C_{\mu}=2 \epsilon C_{\mu}$.

Then one can check that also the interaction terms are invariant. An infinitesimal background rescaling acting on $\Delta_{\mathrm{FP} \mu}{ }^{\nu} C_{\nu}$, as written in (2.24), only affects on the terms $\delta_{C}^{(Q)} \mathbf{X}$. Since $\mathbf{X}$ transforms by a constant shift, $\delta(a d \mathbf{X})=0$. Then, because everything is linear in $C$,

$\delta\left(\overline{\mathbf{g}}^{-1} \mathcal{L}_{C} \overline{\mathbf{g}}+\mathcal{L}_{C} e^{\mathbf{X}} e^{-\mathbf{X}}\right)=-\epsilon\left(\overline{\mathbf{g}}^{-1} \mathcal{L}_{C} \overline{\mathbf{g}}+\left(\mathcal{L}_{C} e^{\mathbf{X}}\right) e^{-\mathbf{X}}\right)$

and the remaining transformations involving $\mathbf{X}$ cancel. Notice the minus sign: this is due to the fact that the Lie derivatives involve the contravariant field $C^{\mu}=\bar{g}^{\mu \nu} C_{v}$, whose transformation is $\delta C^{\mu}=-\epsilon C^{\mu}$. So, finally

$\delta \Delta_{\mathrm{FP} \mu}{ }^{v} C_{v}=-\epsilon \Delta_{\mathrm{FP} \mu}{ }^{v} C_{\nu}$.

This, together with $\delta Y^{\mu \nu}=-d \epsilon Y^{\mu \nu}$ implies that the full ghost action is invariant.

The gauge-fixed action must also contain a term

$S_{\text {aux }}=\int \mathrm{d} x \sqrt{\bar{g}} B_{\mu} Y^{\mu v} B_{v}$,

where $B_{\mu}$ is an auxiliary bosonic field [31]. This Gaussian integral has the effect of removing the determinants of $Y$ from the effective action. Scale invariance is achieved provided the auxiliary field is inert: $\delta B_{\mu}=0$.

We note that the procedure proposed here is by no means unique. If one is interested mainly in the application of the formalism to $f(R)$ theories [39,42-49], where one normally

${ }^{1}$ A factor 1/2 is missing in Eq. (III.18) in [24]. considers a spherical background, then one could define $Y^{\mu \nu}=\bar{R}^{\frac{d-2}{2}} \bar{g}^{\mu \nu}$. This achieves scale invariance without having to introduce an auxiliary field, but it would not work on a flat background. One could also have a mix of $\bar{\Delta}$ and $\bar{R}$, provided the overall power is $\frac{d-2}{2}$. Yet another choice would be the "physical gauge" advocated in [24]. In this case one would just set $h^{\perp}=0$ and $\xi_{\mu}=0$, where $\xi_{\mu}$ is the spinone degree of freedom of $h_{\mu \nu}$. Since $h^{\perp}$ is invariant and $\xi_{\mu}$ trasforms homogeneously under scaling, these conditions are scale invariant. They produce Faddeev-Popov determinants that can be taken care of by introducing suitable auxiliary fields.

\subsection{Cutoff term}

Next we consider the cutoff term, which lies at the root of the issue. It has the general structure

$\Delta S_{k}\left(h_{\mu \nu} ; \bar{g}_{\mu \nu}\right)=\frac{1}{2} \int d^{d} x \sqrt{\bar{g}} h_{\mu \nu} \mathcal{R}_{k}^{\mu \nu \rho \sigma} h_{\rho \sigma}$,

where $\mathcal{R}_{k}^{\mu \nu \rho \sigma}(\bar{\Delta})$, in coordinate space, is a two-point kernel. It is typically chosen to have the form

$$
\begin{aligned}
& \mathcal{R}_{k}^{\mu \nu \rho \sigma}(\bar{\Delta}) \\
& \quad=\frac{1}{2}\left(\bar{g}^{\mu \rho} \bar{g}^{\nu \sigma}+\bar{g}^{\mu \sigma} \bar{g}^{\nu \rho}+a \bar{g}^{\mu \nu} \bar{g}^{\rho \sigma}\right) c k^{d-2} R_{k}(\bar{\Delta})
\end{aligned}
$$

where $a$ and $c$ are dimensionless constants and $R_{k}(0)=k^{2}$, with $k$ the IR cutoff scale which controls the coarse-graining procedure. Usually one defines the RG "time" as $t \sim \ln k$. By dimensional analysis

$R_{k}(\bar{\Delta})=k^{2} r(y), \quad y=\bar{\Delta} / k^{2}$,

where $r$ is a dimensionless function that goes rapidly to zero for $y>1$ and $r(0)=1$.

In the Einstein-Hilbert truncation and in de Donder gauge it is very convenient to choose $a=-1$, so that the tensor structure matches the one of the Hessian (including the gauge-fixing term). Furthermore, it is almost always assumed that $c k^{d-2}=1 /(16 \pi G)$. Then the cutoff combines seamlessly with the Hessian resulting simply in the substitution of $\bar{\Delta} \rightarrow \bar{\Delta}+R_{k}(\bar{\Delta})$, where in this specific case $\bar{\Delta}=-\bar{\nabla}^{2}$. We are not committed to using any specific form of the action here, so we leave the constants $a$ and $c$ unspecified. Such a cutoff is then called "pure" to emphasize that it does not contain any running coupling. 
Similarly one introduces the cutoff operator for the ghosts. Using the decomposition (2.8) we can write

$$
\begin{aligned}
& \Delta S_{k}\left(h_{\mu \nu}^{T}, h ; \bar{g}_{\mu \nu}\right)= \frac{1}{2} \int d^{d} x \sqrt{\bar{g}}\left[h_{\mu \nu}^{T} \bar{g}^{\mu \rho} \bar{g}^{\nu \sigma} \mathcal{R}_{k}^{T}(\bar{\Delta}) h_{\rho \sigma}^{T}\right. \\
&\left.+h \mathcal{R}_{k}(\bar{\Delta}) h\right], \\
& \Delta S_{k}^{\mathrm{gh}}\left(C_{\mu}^{*}, C_{\mu} ; \bar{g}_{\mu \nu}\right)=\int d^{d} x \sqrt{\bar{g}} C_{\mu}^{*} \bar{g}^{\mu \nu} \mathcal{R}_{k}^{\mathrm{gh}}(\bar{\Delta}) C_{\nu} \\
& \Delta S_{k}^{\mathrm{aux}}\left(B_{\mu} ; \bar{g}_{\mu \nu}\right)=\int d^{d} x \sqrt{\bar{g}} B_{\mu}^{*} \bar{g}^{\mu \nu} \mathcal{R}_{k}^{\mathrm{aux}}(\bar{\Delta}) B_{\nu},
\end{aligned}
$$

where $\mathcal{R}_{k}^{T}=c k^{d-2} R_{k}=c k^{d} r(y), \mathcal{R}_{k}=c_{0} k^{d-2} R_{k}=$ $c_{0} k^{d} r(y)$ with $c_{0}=c \frac{2+a d}{2 d}, \mathcal{R}_{k}^{\mathrm{gh}}(\bar{\Delta})=c_{\mathrm{gh}} k^{d-2} R_{k}(\bar{\Delta})=$ $c_{\text {gh }} k^{d} r(y)$ and $\mathcal{R}_{k}^{\text {aux }}(\bar{\Delta})=c_{\text {aux }} k^{d-4} R_{k}(\bar{\Delta})=c_{\text {aux }} k^{d-2} r(y)$.

The Laplacian transforms under background rescalings as in (2.17). Since $k$ does not change under a variation of the background metric, we find from (2.32) $\delta \mathcal{R}_{k}=-2 \epsilon k^{d} y r^{\prime}$. On the other hand $\partial_{t} \mathcal{R}_{k}=d k^{d} r-2 k^{d} y r^{\prime}$, so

$\delta \mathcal{R}_{k}=\epsilon\left(-d \mathcal{R}_{k}+\partial_{t} \mathcal{R}_{k}\right)$

The kernel $\mathcal{R}_{k}^{T}$ transforms in the same way. The remarkable fact is that the first term on the r.h.s. exactly cancels the variation of the volume element. Since the tracefree fluctuation transforms homogeneously, in the same way as the covariant metric, the variations of the inverse metric and those of the fields $h_{\mu \nu}^{T}$ also cancel. Thus the variation of (2.33) is

$$
\begin{aligned}
\delta \Delta S_{k}\left(h_{\mu \nu}^{T}, h ; \bar{g}_{\mu \nu}\right)= & \frac{1}{2} \epsilon \int d^{d} x \sqrt{\bar{g}}\left[h_{\mu \nu}^{T} \bar{g}^{\mu \rho} \bar{g}^{\nu \sigma} \partial_{t} \mathcal{R}_{k}^{T} h_{\rho \sigma}^{T}\right. \\
& \left.+h \partial_{t} \mathcal{R}_{k} h\right]-2 d \epsilon \int d^{d} x \sqrt{\bar{g}} \mathcal{R}_{k} h,
\end{aligned}
$$

where the last term comes from the variation of the trace fluctuation $h$.

The ghost cutoff kernel $\mathcal{R}_{k}^{\text {gh }}$ also transforms as in (2.36) so that

$\delta \Delta S_{k}^{\mathrm{gh}}\left(C_{\mu}^{*}, C_{\mu} ; \bar{g}_{\mu \nu}\right)=\epsilon \int d^{d} x \sqrt{\bar{g}} C_{\mu}^{*} \bar{g}^{\mu \nu} \partial_{t} \mathcal{R}_{k}^{\mathrm{gh}} C_{\nu}$.

Finally, the variation of the auxiliary term works a bit differently. Instead of (2.36) one has

$$
\delta \mathcal{R}_{k}^{\mathrm{aux}}=\epsilon\left(-(d-2) \mathcal{R}_{k}^{\mathrm{aux}}+\partial_{t} \mathcal{R}_{k}^{\mathrm{aux}}\right) .
$$

The first term exactly cancels the transformation due to the measure and inverse metric, so that again

$$
\delta \Delta S_{k}^{\mathrm{aux}}\left(B_{\mu} ; \bar{g}_{\mu \nu}\right)=\epsilon \int d^{d} x \sqrt{\bar{g}} B_{\mu} \bar{g}^{\mu \nu} \partial_{t} \mathcal{R}_{k}^{\mathrm{aux}} B_{\nu}
$$

We note that in comparison with [19] all the terms proportional to $\Delta S_{k}$ itself, which came with a factor $d-6$, are absent here.

\section{The Ward identity}

We now have all the ingredients that are needed to derive the Ward identity. The effective average action (EAA) is defined by

$$
\begin{aligned}
\Gamma_{k}\left(\bar{h}_{\mu \nu}^{T}, \bar{h}, \bar{C}_{\mu}^{*}, \bar{C}_{\mu}, \bar{B}_{\mu} ; \bar{g}_{\mu \nu}\right) \\
=-W_{k}\left(j_{T}^{\mu \nu}, j, J_{*}^{\mu}, J^{\mu}, K^{\mu} ; \bar{g}_{\mu \nu}\right) \\
\quad+\int d^{d} x\left(j_{T}^{\mu \nu} \bar{h}_{\mu \nu}^{T}+j \bar{h}+J_{*}^{\mu} \bar{C}_{\mu}^{*}+J^{\mu} \bar{C}_{\mu}+K^{\mu} \bar{B}_{\mu}\right) \\
\quad-\Delta S_{k}\left(\bar{h}_{\mu \nu}^{T}, \bar{h} ; \bar{g}_{\mu \nu}\right) \\
\quad-\Delta S_{k}^{\mathrm{gh}}\left(\bar{C}_{\mu}^{*}, \bar{C}_{\mu} ; \bar{g}_{\mu \nu}\right)-\Delta S_{k}^{\mathrm{aux}}\left(\bar{B}_{\mu} ; \bar{g}_{\mu \nu}\right),
\end{aligned}
$$

where $W_{k}$ is the generating functional of connected Green functions, $\bar{h}_{\mu \nu}^{T}, \bar{h}$ etc. denote here the classical VEVs of the corresponding quantum fields, the sources $j_{T}^{\mu \nu}, j, J_{*}^{\mu}, J^{\mu}$ and $K^{\mu}$ have to be interpreted as usual as functionals of these classical fields and the last three term subtracts the cutoff that had been added in the beginning to the bare action.

The modified Ward identity for $\Gamma_{k}$ can be obtained as in [19] by first varying $W_{k}$ and then using the Legendre transform. Alternatively, we can start from the integro-differential functional equation

$$
\begin{aligned}
e^{-\Gamma_{k}}\left(\bar{h}_{\mu \nu}^{T}, \bar{h}, \bar{C}_{\mu}^{*}, \bar{C}_{\mu}, \bar{B}_{\mu} ; \bar{g}_{\mu \nu}\right) \\
=\int D h^{T} D h D C^{*} D C D B \operatorname{Exp}\left[-S-S_{\mathrm{GF}}-S_{\mathrm{gh}}-S_{\mathrm{aux}}\right] \\
\quad \times \operatorname{Exp} \int\left[\frac{\delta \Gamma_{k}}{\delta \bar{h}^{T}}\left(h^{T}-\bar{h}^{T}\right)+\frac{\delta \Gamma_{k}}{\delta \bar{h}}(h-\bar{h})+\frac{\delta \Gamma_{k}}{\delta C}(C-\bar{C})\right. \\
\left.\quad+\frac{\delta \Gamma_{k}}{\delta \bar{C}^{*}}\left(C^{*}-\bar{C}^{*}\right)+\frac{\delta \Gamma_{k}}{\delta B}(B-\bar{B})\right] \\
\quad \times \operatorname{Exp}^{*}\left[-\Delta S_{k}\left(h^{T}-\bar{h}^{T}, h-\bar{h} ; \bar{g}\right)\right. \\
\quad-\Delta S_{k}^{\mathrm{gh}}\left(C^{*}-\bar{C}^{*}, C-\bar{C} ; \bar{g}\right) \\
\left.\quad-\Delta S_{k}^{\mathrm{aux}}(B-\bar{B} ; \bar{g})\right]
\end{aligned}
$$

where a bar over a field denotes its vacuum expectation value and we have suppressed all indices for typographical clarity. Varying both sides, we have

$$
\begin{aligned}
\delta \Gamma_{k}= & -\int \frac{\delta \Gamma_{k}}{\delta \bar{h}^{T}}\left\langle\delta h^{T}-\delta \bar{h}^{T}\right\rangle-\int \frac{\delta \Gamma_{k}}{\delta \bar{h}}\langle\delta h-\delta \bar{h}\rangle \\
& -\int \frac{\delta \Gamma_{k}}{\delta \bar{C}}\langle\delta C-\delta \bar{C}\rangle-\int \frac{\delta \Gamma_{k}}{\delta \bar{C}^{*}}\left\langle\delta C^{*}-\delta \bar{C}^{*}\right\rangle \\
& -\int \frac{\delta \Gamma_{k}}{\delta \bar{B}}\langle\delta B-\delta \bar{B}\rangle \\
& +\left\langle\delta \Delta S_{k}\left(h^{T}-\bar{h}^{T}, h-\bar{h} ; \bar{g}\right)\right\rangle+\left\langle\delta \Delta S_{k}^{\mathrm{gh}}(C-\bar{C} ; \bar{g})\right\rangle
\end{aligned}
$$




$$
+\left\langle\Delta S_{k}^{\text {aux }}(B-\bar{B} ; \bar{g})\right\rangle .
$$

The variations (2.10) and those of the ghost and auxiliary fields are at most linear in the fields. Thus $\langle\delta \phi\rangle=\delta \bar{\phi}$ for all fields. All the terms in the first two lines are therefore zero and the only anomalous contribution comes from the cutoff terms. Using the variation in Eqs. (2.37), (2.38), and (2.40) one finds

$$
\begin{aligned}
\delta \Gamma_{k}= & \left\langle\delta \Delta S_{k}\left(h^{T}-\bar{h}^{T}, h-\bar{h} ; \bar{g}\right)\right\rangle+\left\langle\delta \Delta S_{k}^{\mathrm{gh}}(C-\bar{C} ; \bar{g})\right\rangle \\
& +\left\langle\delta \Delta S_{k}^{\mathrm{aux}}(B-\bar{B} ; \bar{g})\right\rangle \\
= & \epsilon\left[\frac{1}{2} \operatorname{Tr}\left(\frac{\delta^{2} \Gamma_{k}}{\delta \bar{h}^{T} \delta \bar{h}^{T}}+\mathcal{R}_{k}^{T}\right)^{-1} \partial_{t} \mathcal{R}_{k}^{T}\right. \\
& +\frac{1}{2} \operatorname{Tr}\left(\frac{\delta^{2} \Gamma_{k}}{\delta \bar{h} \delta \bar{h}}+\mathcal{R}_{k}\right)^{-1} \partial_{t} \mathcal{R}_{k} \\
& -\operatorname{Tr}\left(\frac{\delta^{2} \Gamma_{k}}{\delta \bar{C}^{*} \delta \bar{C}}+\mathcal{R}_{k}^{\mathrm{gh}}\right)^{-1} \partial_{t} \mathcal{R}_{k}^{\mathrm{gh}} \\
& \left.+\frac{1}{2} \operatorname{Tr}\left(\frac{\delta^{2} \Gamma_{k}}{\delta \bar{B} \delta \bar{B}}+\mathcal{R}_{k}^{\mathrm{aux}}\right)^{-1} \partial_{t} \mathcal{R}_{k}^{\mathrm{aux}}+\cdots\right] .
\end{aligned}
$$

Apart from the factor $\epsilon$, the r.h.s. is identical to the r.h.s. of the exact RG equation. (The ellipses stand for terms involving mixed functional derivatives that are present in the exact equation but are neglected in common approximations.) The 1.h.s. of the identity is the total variation of $\Gamma_{k}$, with $k$ held fixed, which can be expressed as

$$
\begin{aligned}
& \delta \Gamma_{k}=\epsilon \int d^{d} x \\
& {\left[2 \bar{g}_{\mu \nu} \frac{\delta \Gamma_{k}}{\delta \bar{g}_{\mu \nu}}+2 h_{\mu \nu}^{T} \frac{\delta \Gamma_{k}}{\delta h_{\mu \nu}^{T}}-2 d \frac{\delta \Gamma_{k}}{\delta h}+2 C_{\mu} \frac{\delta \Gamma_{k}}{\delta C_{\mu}}\right] .}
\end{aligned}
$$

Here and in the following we omit the bars over $h_{\mu \nu}^{T}$ and $h$, since the argument of $\Gamma_{k}$ are always the classical expectation values and no confusion can arise.

\section{The Ward identity and the flow equation}

We have arrived at a remarkably simple result: the anomalous variation in the background scale Ward identity is exactly the "beta functional" of the theory induced by the coarsegraining procedure, as expressed by the r.h.s. of the RG equation:

$\delta_{\epsilon} \Gamma_{k}=\epsilon \partial_{t} \Gamma_{k}$,

where we recall that the variation on the 1.h.s. involves only the functional arguments of $\Gamma_{k}$ and leaves $k$ fixed. Bringing the r.h.s. to the 1.h.s. we obtain

$$
\begin{aligned}
& \int d^{d} x\left[2 \bar{g}_{\mu \nu} \frac{\delta \Gamma_{k}}{\delta \bar{g}_{\mu \nu}}+2 h_{\mu \nu}^{T} \frac{\delta \Gamma_{k}}{\delta h_{\mu \nu}^{T}}-2 d \frac{\delta \Gamma_{k}}{\delta h}+2 C_{\mu} \frac{\delta \Gamma_{k}}{\delta C_{\mu}}\right] \\
& -k \frac{d \Gamma_{k}}{d k}=0 .
\end{aligned}
$$

This is just the statement that the EAA is invariant under scalings of the background metric, accompanied by suitable transformations of the other fields and by a rescaling of the cutoff $k$ :

$\delta k=-\epsilon k$.

As discussed in [19], (4.2) can be solved using the method of characteristics. One must have

$$
\begin{aligned}
\frac{\mathrm{d} \bar{g}_{\mu \nu}}{\mathrm{d} \lambda}=2 \bar{g}_{\mu \nu} ; \quad \frac{\mathrm{d} h_{\mu \nu}^{T}}{\mathrm{~d} \lambda}=2 h_{\mu \nu}^{T} ; \\
\frac{\mathrm{d} \underline{h}}{\mathrm{~d} \lambda}=-2 d ; \quad \frac{\mathrm{d} C_{\mu}}{\mathrm{d} \lambda}=2 C_{\mu} ; \quad \frac{\mathrm{d} k}{\mathrm{~d} \lambda}=-k,
\end{aligned}
$$

whose solutions are simply

$$
\begin{aligned}
\bar{g}_{\mu \nu}(\lambda) & =e^{2 \lambda} \bar{g}_{\mu \nu}(0) ; \quad h_{\mu \nu}^{T}(\lambda)=e^{2 \lambda} h_{\mu \nu}^{T}(0) ; \\
\underline{h}(\lambda) & =\underline{h}(0)-2 d \lambda ; \\
C_{\mu}(\lambda) & =e^{2 \lambda} C_{\mu}(0) ; \quad k(\lambda)=e^{-\lambda} k(0),
\end{aligned}
$$

while $h^{\perp}, C_{\mu}^{*}$ and $B_{\mu}$ are constant. The last relation implies that $\lambda=-t$. Thus the scaling parameter can be identified with the RG time. The combinations

$$
\begin{gathered}
\hat{k}=e^{-\underline{h} / 2 d} k ; \quad \hat{g}_{\mu \nu}=e^{\underline{h} / d} \bar{g}_{\mu \nu} ; \quad \hat{h}_{\mu \nu}^{T}=e^{\underline{h} / d} h_{\mu \nu}^{T} ; \\
h^{\perp} ; \quad \hat{C}_{\mu}=e^{\underline{\underline{h}} / d} C_{\mu}
\end{gathered}
$$

are invariant. ${ }^{2}$ The solution of the Ward identity is therefore a functional

$$
\begin{aligned}
& \Gamma_{k}\left(h_{\mu \nu}^{T}, h^{\perp}, \underline{h}, C_{\mu}^{*}, C_{\mu}, B_{\mu} ; \bar{g}_{\mu \nu}\right) \\
& \quad=\hat{\Gamma}_{\hat{k}}\left(\hat{h}_{\mu \nu}^{T}, h^{\perp}, C_{\mu}^{*}, \hat{C}_{\mu}, B_{\mu} ; \hat{g}_{\mu \nu}\right) .
\end{aligned}
$$

As expected the Ward identity eliminates the dependence of the EAA on the variable $\underline{h}$ and on the total volume of the background metric, replacing it by the dependence on the total volume of the metric $\hat{g}_{\mu \nu}$. The solution can be written entirely in terms of quantities that are invariant under constant Weyl rescaling of the background. In particular, if one specializes to the case when $h^{\perp}=0, h_{\mu \nu}^{T}=0, C_{\mu}^{*}=0$, $C_{\mu}=0, B_{\mu}=0$ one has

$\Gamma_{k}\left(\underline{h} ; \bar{g}_{\mu \nu}\right)=\hat{\Gamma}_{\hat{k}}\left(\hat{g}_{\mu \nu}\right)$

Note that if we set $h_{\mu \nu}^{T}=0$ and $h^{\perp}=0$, then $\hat{g}_{\mu \nu}$ is the classical value of the full quantum metric; see (2.13).

\footnotetext{
$\overline{{ }^{2} \text { Alternatively }}$ one could also define $\hat{k}=\bar{V}^{1 / d} k$, where $\bar{V}$ is the volume in the metric $\bar{g}_{\mu \nu}$.
} 
If we were able to solve the full Ward identities related to arbitrary deformations of the background, we would obtain a functional $\hat{\Gamma}_{\hat{k}}\left(\hat{g}_{\mu \nu}\right)$ that would satisfy a flow equation containing its second derivatives with respect to $\hat{g}_{\mu \nu}$. Having only partly transferred the field dependence from the fluctuation field to $\hat{g}_{\mu \nu}$, we will have a flow equation containing second derivatives with respect to the remaining fluctuation fields and second derivatives with respect to those deformations of $\hat{g}_{\mu \nu}$ that have become dynamical as a result of solving the Ward identity. (In the present case, this is just the overall scale of $\hat{g}_{\mu \nu}$.) This distinction obviously gets blurred when one uses the single-metric approximation.

\section{Discussion}

The main outcome of this paper is the generalization of the results of [19] for the background scale Ward identity in quantum gravity. Morris was able to show that in six dimensions the violation of background scale invariance is given exactly by the r.h.s. of the RG equation. This is reminiscent of the statement that in a classically scale-invariant quantum field theory in flat space, such as massless QCD, the violation of scale invariance is proportional to the beta functions. The physical meaning of the identity is different in the two cases: in QCD it is a genuine anomaly, whereas in quantum gravity the anomalous variation under a change of background can be absorbed by a change of the fluctuation field $\underline{h}$ and of the cutoff $k$, as we have seen in the preceding section. Nevertheless, the two statements are formally the same, and one would expect such general statements to be true in any dimension. Indeed we have shown here that this is the case.

To get this result, however, one has to make certain choices that minimize the breaking of scale invariance. The main difference with [19] is the use of the exponential parametrization for the metric (1.3). When the linear split (1.1) is used, invariance of the full metric requires that the fluctuation field has a transformation opposite to the one of the background field, with the exception of the trace that has a mixed transformation consisting of a homogeneous and an inhomogeneous term. With the exponential parametrization, the fluctuation field transforms in the same way as the background metric, with the exception of the trace that transforms purely by a shift, in much the same way as a dilaton. These transformation rules merely reflect the dimensions of the fields (when the coordinates are dimensionless and a metric has given dimension of area) and the remaining choices also follow the dimensions of each field. The other differences are in the gauge-fixing and cutoff terms: one has to make sure that these do not contain dimensionful couplings that would introduce additional unwanted scale-breaking terms. Of course, it is unavoidable to break background scale invariance by introducing the cutoff scale $k$, but the main point of the present exercise has been to show that if this is the only source of scale-breaking, its effect is entirely contained in the RG flow of the couplings. To this effect, in $d>2$ we have used a higher-derivative cutoff, such as is used in higher-derivative gravity, and a "pure" cutoff, which does not contain any Lagrangian parameter. We stress that with this gauge fixing we are able to prove invariance of the ghost action including all ghost interactions. There may be other procedures that also work well, but these three choices are sufficient to ensure that the Ward identity does not contain additional, unnecessary anomalous terms.

The Ward identity can be used to reduce the number of variables that the effective average action depends upon. Ultimately one would like to reduce the flow equation for $\Gamma_{k}\left(h_{\mu \nu} ; \bar{g}_{\mu \nu}\right)$ to a flow equation for a functional of a single field $\hat{\Gamma}_{\hat{k}}\left(\hat{g}_{\mu \nu}\right)$. Reference [19] and the present work are first steps towards background independence: we have shown how to eliminate from the RG flow the dependence on a single real degree of freedom: the overall scale of the background. This may look like a rather small step, but without it the beta functions are likely to contain spurious terms. We plan to investigate this in concrete calculations. The main value of the present work may lie in restricting the freedom of choice of parametrization, gauge and cutoff scheme. Equation (4.1) is an important statement, even if restricted to constant Weyl transformations: it is expected of any quantum field theory that is invariant under rescalings of the background metric at the classical level. One should be wary of using parametrizations and/or cutoff schemes that violate it.

Acknowledgements G.P.V. thanks Jan M. Pawlowski for interesting discussions.

Open Access This article is distributed under the terms of the Creative Commons Attribution 4.0 International License (http://creativecomm ons.org/licenses/by/4.0/), which permits unrestricted use, distribution, and reproduction in any medium, provided you give appropriate credit to the original author(s) and the source, provide a link to the Creative Commons license, and indicate if changes were made.

Funded by $\mathrm{SCOAP}^{3}$.

\section{References}

1. M. Reuter, Nonperturbative evolution equation for quantum gravity. Phys. Rev. D 57, 971 (1998). arXiv:hep-th/9605030

2. D.F. Litim, J.M. Pawlowski, Wilsonian flows and background fields. Phys. Lett. B 546, 279 (2002). arXiv:hep-th/0208216

3. D.F. Litim, J.M. Pawlowski, Renormalization group flows for gauge theories in axial gauges. JHEP 0209, 049 (2002). arXiv:hep-th/0203005

4. E. Manrique, M. Reuter, Bimetric truncations for quantum einstein gravity and asymptotic safety. Ann. Phys. 325, 785 (2010). arXiv:0907.2617 [gr-qc]

5. E. Manrique, M. Reuter, F. Saueressig, Matter induced bimetric actions for gravity. Ann. Phys. 326, 440 (2011). arXiv:1003.5129 [hep-th] 
6. E. Manrique, M. Reuter, F. Saueressig, Bimetric renormalization group flows in quantum Einstein gravity. Ann. Phys. 326, 463-485 (2011). arXiv:1006.0099 [hep-th]

7. D. Becker, M. Reuter, En route to background independence: broken split-symmetry, and how to restore it with bi-metric average actions. Ann. Phys. 350, 225 (2014). arXiv:1404.4537 [hep-th]

8. I. Donkin, J.M. Pawlowski, The phase diagram of quantum gravity from diffeomorphism-invariant RG-flows. arXiv:1203.4207 [hepth]

9. A. Codello, G. D'Odorico, C. Pagani, Consistent closure of RG flow equations in quantum gravity. Phys. Rev. D 89(8), 081701 (2014). arXiv: 1304.4777 [gr-qc]

10. P. Donà, A. Eichhorn, R. Percacci, Matter matters in asymptotically safe quantum gravity. Phys. Rev. D 89(8), 084035 (2014). arXiv: 1311.2898 [hep-th]

11. N. Christiansen, B. Knorr, J. Meibohm, J.M. Pawlowski, M. Reichert, Local quantum gravity. arXiv:1506.07016 [hep-th]

12. J. Meibohm, J.M. Pawlowski, M. Reichert, Asymptotic safety of gravity-matter systems. arXiv:1510.07018 [hep-th]

13. J.A. Dietz, T.R. Morris, Background independent exact renormalization group for conformally reduced gravity. JHEP 1504, 118 (2015). arXiv:1502.07396 [hep-th]

14. P. Labus, T.R. Morris, Z.H. Slade, Background independence in a background dependent renormalization group. Phys. Rev. D 94(2), 024007 (2016). arXiv: 1603.04772 [hep-th]

15. J.A. Dietz, T.R. Morris, Z.H. Slade, Fixed point structure of the conformal factor field in quantum gravity. arXiv:1605.07636 [hepth]

16. M. Safari, G.P. Vacca, Covariant and single-field effective action with the background-field formalism. arXiv:1607.03053 [hep-th]

17. M. Safari, G.P. Vacca, Covariant and background independent functional RG flow for the effective average action. JHEP 1611, 139 (2016). arXiv:1607.02989 [hep-th]

18. C. Wetterich, Gauge invariant flow equation. arXiv:1607.02989 [hep-th]

19. T.R. Morris, Large curvature and background scale independence in single-metric approximations to asymptotic safety. arXiv:1610.03081 [hep-th]

20. H. Kawai, M. Ninomiya, Renormalization group and quantum gravity. Nucl. Phys. B 336, 115 (1990)

21. H. Kawai, Y. Kitazawa, M. Ninomiya, Ultraviolet stable fixed point and scaling relations in (2+epsilon)-dimensional quantum gravity. Nucl. Phys. B 404, 684 (1993). arXiv:hep-th/9303123

22. T. Aida, Y. Kitazawa, J. Nishimura, A. Tsuchiya, Two loop renormalization in quantum gravity near two-dimensions. Nucl. Phys. B 444, 353 (1995). arXiv:hep-th/9501056

23. A. Eichhorn, On unimodular quantum gravity. Class. Quantum Gravity. 30, 115016 (2013). arXiv:1301.0879 [gr-qc]

24. R. Percacci, G.P. Vacca, Search of scaling solutions in scalar-tensor gravity. Eur. Phys. J. C 75, 188 (2015). arXiv:1501.00888 [hep-th]

25. A. Nink, Field parametrization dependence in asymptotically safe quantum gravity. Phys. Rev. D 91(4), 044030 (2015). arXiv: 1410.7816 [hep-th]

26. M. Demmel, A. Nink, Phys. Rev. D 92(10), 104013 (2015). arXiv: 1506.03809 [gr-qc]

27. A. Nink, M. Reuter, The unitary conformal field theory behind 2D Asymptotic Safety. JHEP 1602, 167 (2016). arXiv:1512.06805 [hep-th]

28. A. Codello, G. D'Odorico, Scaling and renormalization in two dimensional quantum gravity, Phys. Rev. D 92(2), 024026 (2015). arXiv: 1412.6837 [gr-qc]

29. H. Gies, B. Knorr, S. Lippoldt, Generalized parametrization dependence in quantum gravity. Phys. Rev. D 92, 084020 (2015). arXiv:1507.08859 [hep-th]
30. N. Ohta, R. Percacci, A.D. Pereira, Gauges and functional measures in quantum gravity I: Einstein theory. JHEP 1606, 115 (2016). arXiv:1605.00454 [hep-th]

31. N. Ohta, R. Percacci, A.D. Pereira, Gauges and functional measures in quantum gravity II: higher derivative gravity, arXiv:1610.07991 [hep-th]

32. A. Eichhorn, The renormalization group flow of unimodular $f(R)$ gravity. JHEP 1504, 096 (2015). arXiv:1501.05848 [gr-qc]

33. P. Labus, R. Percacci, G.P. Vacca, Asymptotic safety in $\mathrm{O}(\mathrm{N})$ scalar models coupled to gravity. Phys. Lett. B 753, 274 (2016). arXiv:1505.05393 [hep-th]

34. N. Ohta, R. Percacci, G.P. Vacca, Flow equation for $f(R)$ gravity and some of its exact solutions. Phys. Rev. D 92(6), 061501 (2015). arXiv:1507.00968 [hep-th]

35. N. Ohta, R. Percacci, G.P. Vacca, Renormalization group equation and scaling solutions for $\mathrm{f}(\mathrm{R})$ gravity in exponential parametrization. Eur. Phys. J. C 76(2), 46 (2016). arXiv:1511.09393 [hep-th]

36. P. Donà, A. Eichhorn, P. Labus, R. Percacci, Asymptotic safety in an interacting system of gravity and scalar matter, Phys. Rev. D 93(4), 044049 (2016). arXiv:1512.01589 [gr-qc] [Erratum: [Phys. Rev. D 93(12), 129904 (2016)]

37. G. de Berredo-Peixoto, I.L. Shapiro, Higher derivative quantum gravity with Gauss-Bonnet term. Phys. Rev. D 71, 064005 (2005). arXiv:hep-th/0412249

38. A. Codello, R. Percacci, Fixed points of higher derivative gravity. Phys. Rev. Lett. 97, 221301 (2006). arXiv:hep-th/0607128

39. A. Codello, R. Percacci, C. Rahmede, Investigating the ultraviolet properties of gravity with a Wilsonian renormalization group equation. Ann. Phys. 324, 414 (2009). arXiv:0805.2909 [hep-th]

40. N. Ohta, R. Percacci, Higher derivative gravity and asymptotic safety in diverse dimensions. Class. Quantum Gravity 31, 015024 (2014). arXiv:1308.3398 [hep-th]

41. G. Narain, R. Percacci, On the scheme dependence of gravitational beta functions. Acta Phys. Polon. B 40, 3439 (2009). arXiv:0910.5390 [hep-th]

42. A. Codello, R. Percacci, C. Rahmede, Ultraviolet properties of $f(R)$-gravity. Int. J. Mod. Phys. A 23, 143 (2008). arXiv:0705.1769 [hep-th]

43. D. Benedetti, F. Caravelli, The Local potential approximation in quantum gravity, JHEP 1206, 017 (2012). arXiv:1204.3541 [hepth]. [Erratum-ibid. 1210, 157 (2012)]

44. D. Benedetti, On the number of relevant operators in asymptotically safe gravity. Europhys. Lett. 102, 20007 (2013). arXiv:1301.4422 [hep-th]

45. J.A. Dietz, T.R. Morris, Asymptotic safety in the $f(R)$ approximation. JHEP 1301, 108 (2013). arXiv:1211.0955 [hep-th]

46. J.A. Dietz, T.R. Morris, Redundant operators in the exact renormalisation group and in the $f(R)$ approximation to asymptotic safety. JHEP 1307, 064 (2013). arXiv:1306.1223 [hep-th]

47. M. Demmel, F. Saueressig, O. Zanusso, Fixed-functionals of threedimensional quantum Einstein gravity. JHEP 1211, 131 (2012). arXiv: 1208.2038 [hep-th]

48. M. Demmel, F. Saueressig, O. Zanusso, RG flows of quantum Einstein gravity in the linear-geometric approximation. Ann. Phys. 359, 141 (2015). arXiv:1412.7207 [hep-th]

49. M. Demmel, F. Saueressig, O. Zanusso, A proper fixed functional for four-dimensional quantum Einstein gravity. JHEP 1508, 113 (2015). arXiv:1504.07656 [hep-th] 